\title{
ESCALAS DE ANÁLISIS EN GEOARQUEOLOGÍA: APLICACIÓN EN UN VALLE DEL NOROESTE DE ARGENTINA
}

\author{
Maria Marta Sampietro Vattuone a y José Luis Peña Monnéb
}

\begin{abstract}
Resumen
Los estudios geoarqueológicos constituyen un gran aporte frente a otros enfoques para la comprensión de las sociedades humanas del pasado, dada la complejidad que pueden alcanzar en el manejo de múltiples líneas de evidencia. La construcción de un modelo geoarqueológico de una región implica el manejo conjunto de diversas escalas temporales y espaciales, lo cual otorga riqueza a las interpretaciones y una perspectiva coevolutiva de las variables culturales y naturales, muchas veces interpretadas como opuestas y antagónicas. El objetivo de este trabajo, es mostrar, cómo la geoarqueología puede constituir una herramienta de aproximación ágil al conocimiento de las sociedades del pasado, la reconstrucción del paisaje, de los paleoambientes del momento y de la cultura material a través de diversas escalas espacio-temporales de análisis. Estas escalas pueden estructurarse hasta generar un todo integrado y articulado para producir inferencias fiables de procesos que se entrelazan en un continuum espaciotemporal. Para ello se ilustra el proceso con los resultados obtenidos en las últimas décadas de trabajo en el valle de Tafi (Tucumán, Argentina) incluyendo ejemplos de articulación entre la escala regional, escala intermedia y escala de detalle.
\end{abstract}

Palabras clave: modelo geoarqueológico, mapa geomorfológico, metodología, escalas espacio-temporales, valle de Tafi.

Abstract

SCALES OF ANALYSIS IN GEOARCHEOLOGY: APPLICATION IN A VALLEY FROM NORTHWEST ARGENTINA

Geoarchaeological studies constitute a great contribution compared to other approaches for the understanding of past human societies. This is because the complexity that these sorts of studies reach in the management of multiple lines of evidence. The construction of a geoarchaeological model of a region implies the management of various temporal and spatial scales, which gives richness to the interpretations and a coevolutionary perspective of the cultural and natural variables, often interpreted as opposite and antagonistic. The objective of this work is to show how geoarchaeology can constitute a tool for an agile approach to the knowledge of the societies of the past, the reconstruction of the landscape, the paleoenvironments of past times, and the material culture through various spatio-temporal scales analyses. These scales can be structured to generate an integrated and articulated whole to produce reliable inferences of processes that are crisscrossed in a space-time continuum. Finally, the process is illustrated with the results obtained in the last decades of work in the Tafi Valley (Tucuman, Argentina), including examples of articulation between the regional scale, intermediate scale and detail scale.

Keywords: geoarchaeological model geomorphological map, methodology, spatial scale, temporal scale, Tafi valley.

a Laboratorio de Geoarqueología, Facultad de Ciencias Naturales e IML - Universidad Nacional de Tucumán (UNT), CONICET. sampietro@tucbbs.com.ar

bDepartamento de Geografía y Ordenación del Territorio, Universidad de Zaragoza, España. jlpena@unizar.es

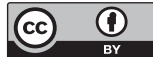




\section{Introducción}

Los estudios geoarqueológicos constituyen un gran aporte frente a otros enfoques para la comprensión de las sociedades humanas del pasado, dada la complejidad que pueden alcanzar en el manejo de múltiples líneas de evidencia (Rapp 2007). Ello es más evidente si están orientados a la confección de modelos geoarqueológicos evolutivos que involucran aspectos de los cambios ambientales naturales y antrópicos en conjunción con los cambios culturales y el uso del territorio (Sampietro Vattuone y Peña Monné 2019a). Pero cada aproximación al conocimiento de un área arqueológica de interés puede realizarse desde múltiples escalas, tanto espaciales como temporales, generando un conjunto multidimensional. Como ocurre por ejemplo con el ambiente físico donde cada unidad de área se vincula a procesos que poseen tiempos propios de cambio generando un complejo entramado que debe ser abordado detalladamente. Este proceso conlleva a explicitar no solo la metodología, sino también las escalas — tanto en espacio como en tiempo- disponibles, que en general son propias de los objetivos que se enfrentan. En palabras de Harris (2006) a menos que estos aspectos sean explícitamente reconocidos en arqueología, los resultados derivados de estudios espaciales carecen de sentido.

A simple vista, parece algo intrínseco al desarrollo de una investigación arqueológica pasar de una escala a otra, en un continuo que abarca desde análisis microscópicos hasta análisis de grandes regiones, incluyendo adaptaciones culturales de distinta índole que ocurrieron hace tiempo atrás. Pero en realidad son pocos los arqueólogos que confrontan la validez de este recurso y controlan en qué medida el manejo de tales dimensiones es adecuado para los datos disponibles y representativo de los procesos (tanto en espacio como en tiempo) que se interpretan (Lock y Molyneaux 2006).

En el caso del desarrollo de modelos geoarqueológicos evolutivos, tales como los propuestos por Sampietro Vattuone y Peńa Monné (2019a), el entramado entre escalas y líneas de evidencia (proxies) es muy complejo. Uno de los grandes problemas interpretativos se deriva de la falta de conocimiento de los mecanismos adecuados para incluir la interpretación de los procesos de baja resolución (temporal y espacial) en la escala de alta resolución humana. Para solventar esto, debe ser rigurosamente establecido qué escala involucra cada uno de los niveles de conocimiento obtenidos y en qué medida son acordes a la información que se reconstruye. Además, los vínculos entre los distintos niveles deben realizarse mediante indicadores válidos para dos o más niveles explicativos diferentes, generando puentes interescalares.

El objetivo de este trabajo es mostrar cómo la geoarqueología puede constituir una herramienta de aproximación ágil al conocimiento de las sociedades del pasado, la reconstrucción del paisaje, de los paleoambientes del momento y de la cultura material a través de diversas escalas espacio-temporales de análisis. Estas escalas pueden estructurarse hasta generar un todo integrado y articulado para producir inferencias fiables de procesos que se entrelazan en un continuum espaciotemporal. Para ello se ilustrará el proceso con los resultados obtenidos en las últimas décadas de trabajo en el valle de Tafí (Tucumán, Argentina).

\section{Las escalas en diversas disciplinas y su uso en geoarqueología}

A primera vista, la concepción de escala espacial está directamente relacionada con la relación de tamaño que existe entre una representación cartográfica (un modelo normalmente en papel y actualmente con frecuencia digital) y el tamańo que tiene el rasgo referido en la realidad. Esta proporción es ampliamente utilizada en el mundo de la geografía (Bird 1989; Mcmaster y Sheppard 2004) y por supuesto en arqueología (Spaulding 1960; Clarke 1977). En un sentido más amplio, la escala es percibida en términos de representación espacio-temporal, experiencia u organización de eventos y procesos (Smith 2000), e incluso como concepto subordinado al significado de las cosas y la relación personal y de otros animales con el mundo en una escala fenomenológica 
individual; affordances en términos del autor hacia un planteo sobre los sistemas perceptuales y la psicología ecológica (Gibson 1977, 1982).

Pero más allá de estas definiciones generales, el movimiento de la información material a datos procesados y finalmente al conocimiento obtenido requiere un análisis más profundo del rol de las escalas — sean temporales y/o espaciales — en la configuración y obtención de tal información, hasta lograr el desarrollo de una línea de tiempo (Lock y Molyneaux 2006).

Uno de los pioneros de la sistematización de las escalas en arqueología fue Spaulding (1960) quien planteó, en los inicios de la arqueología cuantitativa, una equivalencia entre escala y «dimensiones» incluyendo tres: el espacio, el tiempo y la forma; e introduciendo la idea de correlación entre las tres. Posteriormente se introdujeron variables sociales tales como sociedad, comunidad, grupo e individuo (Mathieu y Scott 2004). Pero la mayoría de las exploraciones relacionadas a las escalas en arqueología se orientaron a cuestiones cuantitativas o a cuestiones medianamente cuantificables (Mathieu y Scott 2004).

Haggett (1965) y Harvey (1968) plantearon tres factores que condicionan el manejo de las escalas en geografía (y por extensión en arqueología): (1) cobertura, relacionada con la necesidad de estudiar el mundo en todas las escalas relevantes aplicando técnicas de exploración, re-prospección y muestreo; (2) estandarización de las escalas, suma y valoración de relevancia de procedimientos para obtener los datos analíticos necesarios; y (3) vínculos de la escala, vinculado a la necesidad de enlazar los resultados en una relación comparable, de tipo contextual. A estas consideraciones se suma lo que Robinson (1950) y Alker (1969) denominaron falacia ecológica que sugiere que la escala condiciona los patrones que se observan y nuestra habilidad para entender procesos que no son observados.

Estas discusiones y consideraciones sobre el uso y manejo de las escalas no han sido ajenas a la geoarqueología (Stein 1993; Butzer 2008; Denham 2008; Fouache 2013; McNamee et al. 2013; Edwards et al. 2017; entre otros). Stein (1993) destaca que las escalas, tanto en el caso de arqueólogos, geoarqueólogos y geocientíficos, son consideradas por los investigadores en dos momentos: en el tamaño y resolución de los datos descritos, así como en la resolución espacial y temporal de la interpretación, aludiendo al tamaño de los fenómenos observados y a la resolución de las interpretaciones. Dado que se trabaja con disciplinas históricas, sus interpretaciones incorporan resoluciones determinadas por la naturaleza del registro, que se vuelve más fragmentario cuanto más antiguo es, limitando las interpretaciones. Cada una de las disciplinas que involucran el uso de escalas ha establecido cuáles son las escalas adecuadas para interpretación (Webb 1976; Modderman 1988; Schumm 1991). Una de las más jóvenes es la geoarqueología que por su naturaleza ha recurrido a las técnicas de las ciencias de la tierra para realizar interpretaciones sobre el pasado humano en la escala (resolución) definida desde la arqueología. Estas interpretaciones interdisciplinarias son complejas y solo pueden ser resueltas explicitando las escalas y resoluciones de sus interpretaciones (Harris 2006). Butzer (2008) expone que una aproximación multiescalar provee vínculos entre macroescala (paisaje) y microescala (sitio, estrato y depósito) en un proceso denominado scale-switching, de lo grande a lo pequeńo y vuelta atrás, como el corazón de la geoarqueología.

La escala, entonces, es la relación entre el tamaño (y duración) de algo y su representación y por lo tanto es un modelo construido que contiene los elementos que consideramos sobresalientes de esa realidad, sea este un artefacto, un rasgo o un proceso (Zimmerman y Artz 2006). Se acepta que es una aproximación multiescalar mixta, definida como métodos complementarios de escala y resolución variable para incrementar el potencial interpretativo de la interacción hombre/ambiente (Edwards et al. 2017).

Los diseños de investigación suelen carecer de explicitaciones relacionadas con las escalas seleccionadas para llevar a cabo la investigación (Wobst 2006), aunque estas determinarán en muchos casos el patrón inferido en los procesos estudiados (Banning 2002) dado que están ligadas estrecha- 
mente a cuestiones de muestreo y por lo tanto requieren atención metodológica explícita (Burger y Todd 2006). La aplicación de un conjunto preciso de técnicas controlando las variables escalares incrementa la comprensión de la historia de formación de un paisaje permitiendo interpretaciones más precisas sobre el uso de la tierra y los impactos producidos sobre ella por el hombre, incluyendo no sólo las variables del pasado sino las actuales.

Si bien existen muchas definiciones de escala, esta se vincula al concepto de resolución, y no al tamaño, el cual es visto un objeto/proceso. En geografía o fotografía, cuando se trata de pequeña escala implica baja resolución normalmente incluyendo grandes áreas (1:50.000 a 1:1.000.000), escalas intermedias a resoluciones medianas (1:20.000-1:70.000) y pequeńa escala se refiere a imágenes de gran resolución (1:20.000 o menores). A medida que aumenta la resolución de un fenómeno, mayor es su complejidad tanto espacial como temporal. Así, largos períodos de tiempo son considerados de baja resolución, al contrario que los de corta duración (Stein 1993, 2002).

\section{El manejo de las escalas en la construcción de un modelo geoarqueológico}

En este trabajo, para evitar confusiones relativas al empleo de "gran escala/baja resolución» y "pequeña escala/alta resolución" nos referiremos a escala regional (Fig. 1a) para describir grandes áreas de terreno y lapsos temporales; escala intermedia (Fig. 1b) para sitios y procesos de pocos siglos; y escala de detalle (Fig. 1c) para estructuras, fenómenos y procesos más cortos. Consideramos que la selección de las variables explicativas válidas para definir el conjunto de los procesos que operan en la realidad requiere de un método espacio-temporal multiescalar explícito, donde es necesario llenar el vacío que existe entre las escalas a las cuales se construyen los modelos teóricos y las escalas de las unidades de observación adoptadas para colectar datos y la subsiguiente agregación de datos utilizados en la evaluación de estos modelos. Respecto al concepto relativo a que observaciones más detalladas conducen a más resolución en la construcción de los modelos espaciotemporales, consideramos que esto en realidad depende de la relevancia de los datos que aporta la variable considerada en el contexto global de lo que se pretende explicar. En definitiva, la resolución que aporta cualquier variable a un modelo geoarqueológico, depende del peso de la misma en el proceso que se está investigando y se relaciona con su calidad explicativa, independientemente de la resolución intrínseca del dato, derivada de su naturaleza ( $v \cdot g$. el caso de un análisis de pastas cerámicas entra en una escala de detalle, y puede ser muy preciso en su realización, pero no es adecuado para explicar la ocupación de un territorio, aunque la pasta cerámica implique un manejo territorial de recursos).

Propuestas metodológicas previas, desarrolladas a partir de nuestra experiencia en medio áridos, han permitido establecer la importancia de la generación de modelos geoarqueológicos evolutivos que integran evidencias de diversas disciplinas. Estos han sido probadamente aplicados en Europa (Constante et al. 2010, 2011; Pérez-Lambán et al. 2014; Peña-Monné et al. 1996, 2004, 2014, 2018a, 2018b; Peña Monné 2018) y en América (Sampietro-Vattuone y Peña-Monné 2014, 2016; Sampietro-Vattuone et al. 2017, 2018a, 2018b, 2019; Peña-Monné y Sampietro Vattuone 2014, 2018, 2019; Peña-Monné et al. 2013, 2016; Maldonado et al. 2016), logrando integrar aspectos relacionados a cambios climáticos y sus efectos en larga duración con carácter global, media duración con carácter generalmente regional y corta duración de alcance local y normalmente restringidos a eventos climáticos excepcionales, en consonancia con aspectos relacionados con ocupaciones humanas y cultura material de la época (o épocas) analizada.

La construcción de modelos geoarqueológicos parte de (i) la construcción de mapas geomorfológicos que contengan la información arqueológica cartografiable y la identificación de las unidades morfosedimentarias más representativas; seguido de (ii) la descripción de las unidades morfosedimentarias y establecimiento de las relaciones espaciales y evolutivas de las mismas integrando la información arqueológica, cronológica y paleoambiental disponible; a continuación (iii) el estudio 


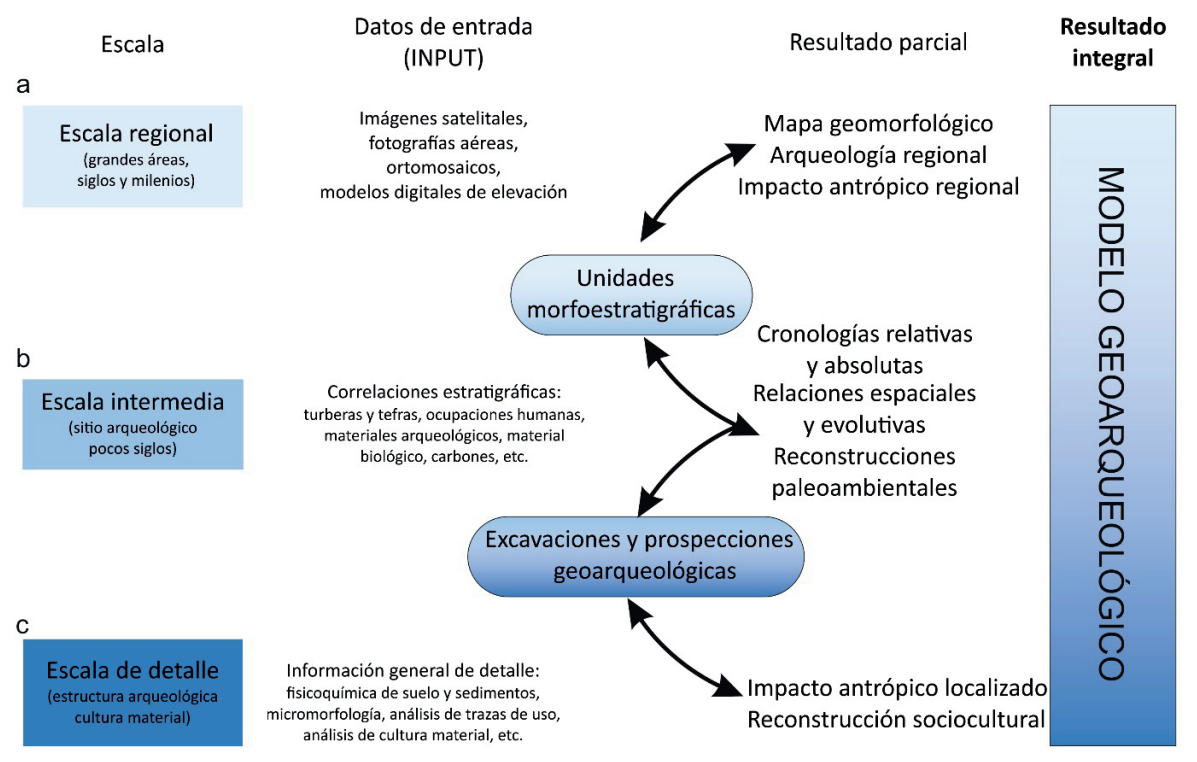

Figura 1. Diagrama general de las escalas de aproximación, datos requeridos para la organización de la información, resultados parciales esperados, información de enlace y resultado final integrado.

pormenorizado de los perfiles longitudinales y transversales obtenidos de diferentes registros de la zona de estudio que integren toda la información, permitiendo además establecer sincronicidades y retardos en la manifestación de los procesos humanos y naturales en las diversas unidades; y finalmente, (iv) la realización de diagramas de síntesis que involucren toda la información disponible a nivel local, regional y global (Sampietro Vattuone y Peña Monné 2019a).

El éxito de esta metodología dependerá de la calidad de la información recuperada y de las posibilidades de establecer relación entre todos los datos recolectados. Tal como está planteada, ofrece sin duda una oportunidad inigualable para lograr esquemas sintéticos comprehensivos, perfectibles conforme avance la investigación. Sin embargo, aquella primera propuesta ha dado por sobreentendido el manejo de las escalas de recolección de datos, la representatividad de los mismos, su precisión y la posibilidad de integración de resultados que ellas ofrecen.

$\mathrm{Al}$ abordar la primera etapa de trabajo, la construcción de mapas geomorfológicos que sirvan de lienzo a la información que luego se detallará en otros planos y procesos de la investigación, partimos de una escala regional (Fig. 1a). La resolución en la recolección de información está condicionada por la escala a la cual tenemos las imágenes de base (satelitales, fotografías aéreas, ortomosaicos, etc.) además de nuestra capacidad para aplicar técnicas de foto-interpretación e identificación visual que constituirán la primera aproximación al objeto de estudio. La realización del mapa geomorfólogico regional de base, conlleva trabajo de campo orientado, no solo, a controlar si los rasgos están correctamente identificados, sino también afinar los procesos involucrados en su formación y complementar la información cartográfica con el registro de las características internas de las unidades geomorfológicas reconocidas. Este trabajo de prospección y control nos invita a afinar la precisión de nuestras apreciaciones y provee indicios que permiten incorporar nueva información de mayor detalle.

En términos temporales, es usual en las representaciones realizadas a esta escala, identificar efectos producto de procesos de larga duración, normalmente de amplitud espacial apreciable a esta resolución. Aunque es posible encontrar rasgos derivados de eventos excepcionales concretos, como v.g. grandes inundaciones, avulsiones, flujos de detritos de magnitud considerable y lluvias de cenizas volcánicas. La extensión de estos últimos dependerá en muchos casos no solo de la 
magnitud del evento, sino también del tiempo transcurrido entre la ocurrencia del mismo y la realización de la investigación. Normalmente, cuanto mayor es el tiempo transcurrido, más fragmentarias en términos espaciales son las evidencias del mismo.

Para el Noroeste Argentino, normalmente las fotografías aéreas más antiguas de mayor cobertura son de 1970, a escala 1:50.000 lo cual, tratándose de un trabajo de características geomorfológicas de detalle a veces son de insuficiente resolución y por lo tanto deben complementarse con imágenes satelitales actuales. Por otra parte, la implementación de técnicas digitales permite realizar ampliaciones específicas (aunque no mejorando la resolución) de zonas de particular interés arqueológico.

La escala a la cual obtendremos nuestro mapa geomorfológico de base poseerá el detalle que nos permitan los medios con los cuales ha sido realizado, y, en tanto modelo cartográfico de la realidad, será producto de una serie de toma de decisiones relacionadas con qué representar y cómo hacerlo, de modo que incluso la calidad gráfica y explicativa deberá ser conscientemente adecuada al sistema de representación y edición posibles. Por otra parte, al servir de mapa de contexto general, podrá contener los rasgos arqueológicos identificables mediante la interpretación de las imágenes de base y la distribución de aquellos conocidos que se quiera integrar, en determinados contextos su representación solo podrá utilizar símbolos identificativos a causa de la escala. En muchos casos, el impacto de las acciones humanas a largo plazo ya puede estar plasmado en esta representación, como es el caso de grandes afecciones en el paisaje producidas de manera sostenida a lo largo de siglos, como el impacto de la agricultura, o de obras hidráulicas que han significado la transformación de grandes áreas.

Por otra parte, durante el trabajo de campo, además de controlar la calidad y ajustar nuestras interpretaciones en gabinete, se puede acceder al conocimiento del interior de las unidades geomorfológicas mediante el estudio de perfiles representativos, contenedores de registros estratigráficos con numerosos indicadores geoarqueológicos y paleoambientales, necesarios para la interpretación regional. Estos registros involucran una escala de análisis compatible con la regional/geomorfológica y la meso escala que involucra procesos y rasgos de cobertura espacial intermedia inherentes a cada unidad geomorfológica específica. Proveen información evolutiva, tanto en términos de proceso (qué y cómo pasó) como temporal (cuándo pasó), de cada unidad estudiada. Permite por lo tanto poner en relación al conjunto de unidades de la región, ordenarlas cronológicamente y conocer qué procesos estuvieron involucrados en su formación en cada etapa. En este punto, la resolución temporal de los procesos estará dada por el contenido de los registros mostrados por los perfiles como consecuencia del ambiente en que se generaron y la mayor o menor presencia antrópica. Así, pueden contener capas de turba, niveles de tefras procedentes de erupciones coetáneas, capas lacustres, fósiles, niveles de carbón, suelos, y sobre todo material arqueológico in situ o transportado. Algunos perfiles pueden proporcionar edades absolutas o relativas para crear un marco cronológico de la unidad morfosedimentaria correspondiente. Otros pueden contener niveles-guía para la correlación estratigráfica entre registros de la misma unidad y su diferenciación con otras unidades. Estos datos son de gran importancia para enriquecer el mapa regional inicial con información cronológica fiable a la vez que integrando información de mayor resolución reflejada en la identificación y el ordenamiento de los diversos procesos identificados en cada perfil y su edad. Así se conforma la información de lo que hemos dado en llamar escala intermedia (Fig. 1b). Las descripciones detalladas morfoestratigráficas constituyen así el vínculo entre los procesos de escala regional y los de escala intermedia.

El enfoque geoarqueológico propuesto para la producción de modelos evolutivos integra en este momento de la investigación una serie de evidencias introducidas por actividad humana o consecuencia de la misma que están vinculadas a lo que ocurre en esta escala de aproximación. Estas evidencias tienen que ver con la intervención humana en el paisaje para su uso y adecuación para diversos fines, como el uso de madera y leña, la creación de pastizales para pastoreo, aterrazamiento 
y roturación de tierras, asentamiento de diversos tipos, actividades extractivas como canteras, etc. Normalmente, a la evidencia material concreta representada por estructuras de terrazas, corrales o residencias se suman acciones tales como incendios intencionales que dejan niveles carbonosos de gran continuidad en los cortes estratigráficos. En general, estas actividades proveen asimismo evidencias que permiten ajustar nuestra escala cronológica y generar una línea de tiempo de mayor detalle involucrada con la formación de las unidades morfoestratigráficas que se describen y sirven, asimismo, de vínculo entre lo que está ocurriendo a escala intermedia y lo que ocurre a escala de detalle en el ámbito del interior de los sitios y estructuras arqueológicas.

Tal como se anticipó, el análisis de mayor resolución de los sitios arqueológicos involucra el trabajo en una escala de detalle (Fig. 1c). Normalmente, los análisis de las superficies de ocupación, se trate del contexto que sea, aportan mucha información en este sentido. Inclusive, mucha información obtenida en contextos agrarios, tal como calidad del suelo, técnicas de manejo, gestión de recursos en términos de acopio y distribución, entre otras, están dando indicios de acciones que promueven impactos de escala intermedia y de detalle (normalmente identificados a partir de indicadores fisicoquímicos). La construcción de una unidad residencial obedece a intereses sociales (individuales y colectivos) que impacta en el ámbito local y de la unidad de paisaje sobre la que se asienta. Lo mismo ocurre en relación a las elecciones relativas a los modos de producción. Así, estudios fisicoquímicos tanto de la cultura material como de los lugares de residencia y producción ofrecen el puente entre la vida cotidiana y los fenómenos de corta duración, altamente localizados espacialmente, y la escala intermedia de interpretación. En términos temporales, aproximaciones a rasgos tales como la vida cotidiana en una unidad residencial, una tumba en el conjunto de un cementerio, el conjunto del utillaje de vinculado a una actividad determinada, el establecimiento de las áreas de actividad en una residencia que obedece a pautas de comprensión íntima del espacio socialmente compartidas, nos provee una ventana acotada en el tiempo de una sociedad cuya precisión en términos de su localización temporal depende del método de datación aplicado y las líneas de evidencia seleccionadas para su análisis. Nuevamente, el entramado final de la información, tanto en cuanto a resolución como precisión, dependerá de la toma de decisiones del investigador y las técnicas aplicadas para la recolección de la información y la habilidad para integrarlas en el modelo final de la realidad que se construya.

Si bien se han discriminado tanto escalas temporales como espaciales, intentando sistematizarlas en tres categorías instrumentales básicas, hay que tener presente que cada proceso representa el transcurso de cierto tiempo que está reflejado por los rasgos que se identifican a través de las diversas técnicas aplicadas. Por otra parte, estos procesos aparecen imbricados entre sí, representando ciclos que se entrelazan en el espacio y reflejan además características ambientales específicas incluyendo cambios climáticos (Burillo y Peña Monné 1984) de largo plazo (normalmente de carácter global), de mediano plazo (especialmente fluctuaciones climáticas del Holoceno de carácter regional) y de corto plazo (eventos climáticos excepcionales que suele ser locales). El reflejo final de estos procesos dinámicos entrelazados se logra mediante el desarrollo de un modelo geoarqueológico (en el sentido propuesto por Sampietro Vattuone y Peña Monné 2019a) que constituye la integración final de todas las variables. El correcto control de las escalas, el conocimiento de su alcance explicativo y del error intrínseco que conlleva la determinación de cada indicador contribuirán a lograr un modelo de funcionamiento de la realidad a lo largo del tiempo.

El mapa geomorfológico de partida constituye una representación gráfica espacio-temporal de procesos acaecidos y otros activos en una región, producto de la evolución ambiental de la misma. Integra fenómenos resultantes de factores naturales y antrópicos. El interior de las unidades geomorfológicas muestra fenómenos de mayor resolución espacial y temporal a través de los contextos morfoestratigráficos que contienen, permitiendo enlazar la escala previa con fenómenos, procesos y acontecimientos de escala intermedia, cuya representación normalmente se realiza en gráficos y esquemas de menor alcance espacial. Finalmente, pequeños sectores, como las unidades 
residenciales, agrarias, etc. poseen información de muy corta duración relacionadas con la vida cotidiana (que suele repetirse a lo largo de períodos de tiempo intermedios) que dejan tras de sí un amplio espectro de evidencias fisicoquímicas y biológicas que sirven de enlace entre las escalas precedentes y las apreciaciones de escala de detalle. En conjunto, esta evidencia espaciotemporal dinamiza las interpretaciones expresadas a través de modelos geoarqueológicos, cuyo alcance es variable según la calidad de la información, su representatividad y el tamaño de la unidad que se está modelando.

\section{El caso del valle de Tafí}

El valle de Tafí se encuentra ubicado al oeste de la provincia de Tucumán, Argentina (Fig. 2). Forma parte de las Sierras Subandinas cuyo basamento es ígneo-metamórfico. Tiene una extensión aproximada de $450 \mathrm{~km}^{2}$ y el fondo de valle se ubica entre los 1800 y $2300 \mathrm{msnm}$. La temperatura media anual es de $13^{\circ} \mathrm{C}$ y las precipitaciones alcanzan hasta los $550 \mathrm{~mm}$ anuales. Hasta la fecha, se han identificado asentamientos pertenecientes al período agroalfarero prehispánico (períodos Formativo [360 a.C-1000 AD] y Desarrollos Regionales [1000-1490 AD]), algunos indicios incaicos (1490-1535 AD) y su temprano ingreso al régimen colonial a partir de 1535, cuando arribaron los españoles a la región (Sampietro Vattuone y Peña Monné 2016).

Las investigaciones geoarqueológicas que se iniciaron en el año 1993, han permitido gradualmente desarrollar el modelo geoarqueológico de la región, representativo también de otras zonas aledañas (Sampietro Vattuone et al. 2018a). El mapa geomorfológico del valle (SampietroVattuone y Peña-Monné 2019b) (Fig. 2), muestra la distribución espacial de las diversas unidades geomorfológicas identificadas en lo que hemos llamado escala regional. La evolución holocena está representada por cuatro unidades agradativas denominadas $\mathrm{H} 1$ a H4 (de la más antigua a la más reciente). La unidad $\mathrm{H} 2$ es la que ocupa mayor superficie en el fondo de valle. Datos cronológicos obtenidos a una escala intermedia de análisis ponen en evidencia que esta unidad se formó contemporáneamente al período agrario prehispánico de la zona.

La información de escala intermedia ha permitido identificar la evolución completa del Holoceno en fases agradativas y degradativas que incluyen niveles guía representados por tefras (Sampietro-Vattuone et al. 2017, 2019) que facilitan las correlaciones estratigráficas (Fig. 3), reafirmando las observaciones realizadas a escala regional, mientras se construía el mapa geomorfológico. A partir de estos niveles se han establecido las relaciones espacio temporales entre las diversas unidades que se identifican en el valle de Tafí (Sampietro-Vattuone y Peña-Monné 2016, 2019b). Además, las dataciones (TL, C-14), la presencia de materiales arqueológicos, suelos, turberas, entre otros rasgos (Fig. 3), han permitido conocer la velocidad a la que actuaron los procesos morfodinámicos durante el periodo de ocupación y el impacto que produjo el cambio de uso del suelo, hacia el sistema productivo agroalfarero en el valle, en una escala de aproximación intermedia, señalada por la respuesta individual de cada unidad geomorfológica (Sampietro-Vattuone et al. 2018a; Peña-Monné y Sampietro-Vattuone 2019).

En definitiva, la extensión que ocupan las unidades $\mathrm{H} 2$ en el mapa es el reflejo geomorfológico producto de los procesos de morfogénesis acelerada, desencadenados por la sobreexplotación del valle durante el período agroalfarero prehispánico (Sampietro-Vattuone y Peńa-Monné 2019b; Sampietro-Vattuone et al. 2019). De acuerdo a la información recuperada, las características del paisaje actual se deben, en gran medida, al efecto de los procesos morfodinámicos acaecidos durante ese período; a esto se suma la intensa ocupación del terreno, tanto con fines residenciales, como de producción mediante la construcción de sistemas de terrazas que aún están presente en la actualidad (muy degradados en algunos sectores).

Reintroduciéndonos en la escala intermedia y transitando hacia una escala de detalle, en la búsqueda de evidencias que den soporte a estas apreciaciones, se ha constatado que los procesos 


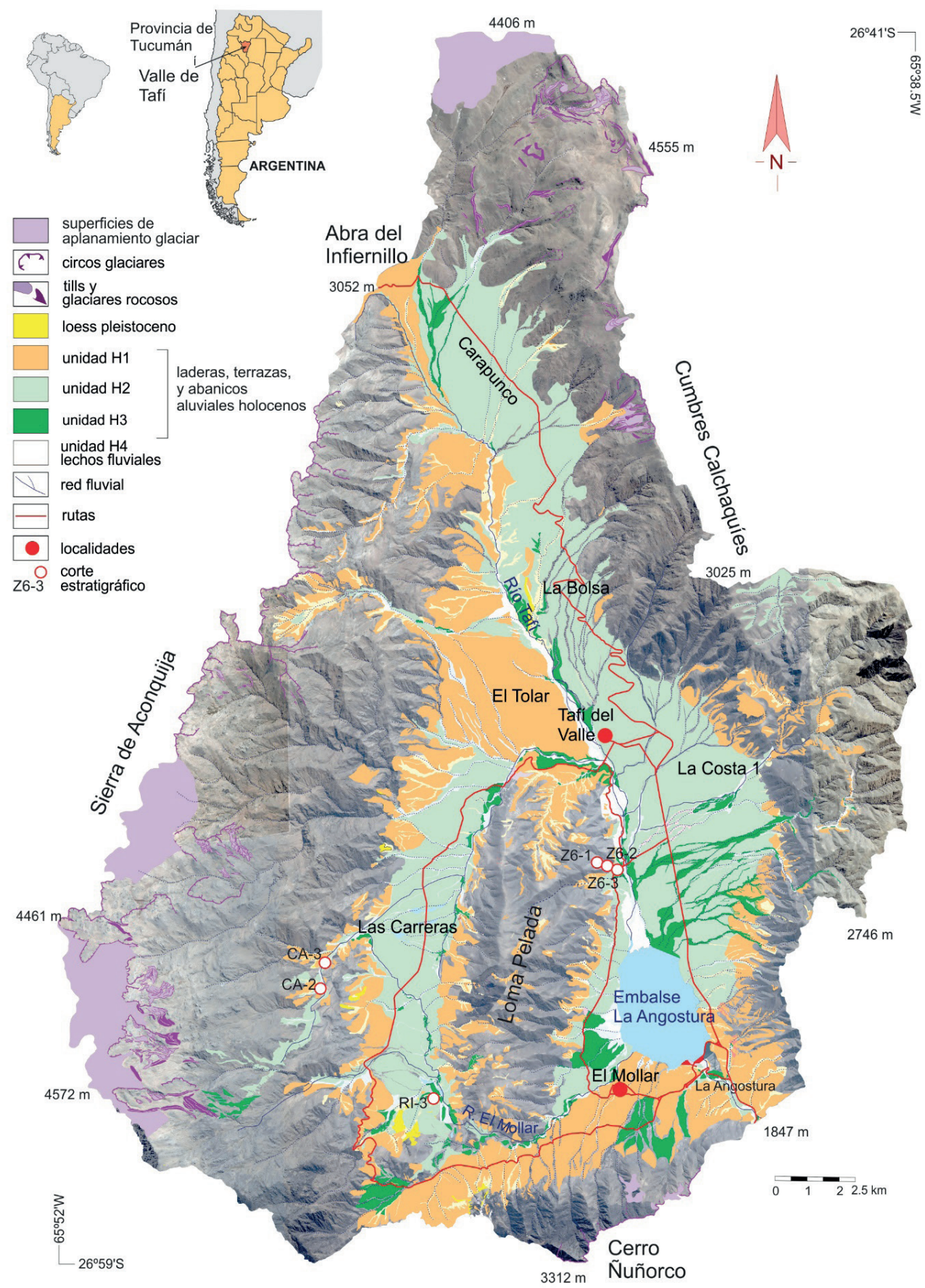

Figura 2. Representación de escala regional. Mapa geomorfológico del valle de Tafí (Provincia de Tucumán, Noroeste Argentino). Nótese la extensión ocupada por la unidad $\mathrm{H2}$, correspondiente cronológicamente al periodo agroalfarero (figura de los autores). 
a
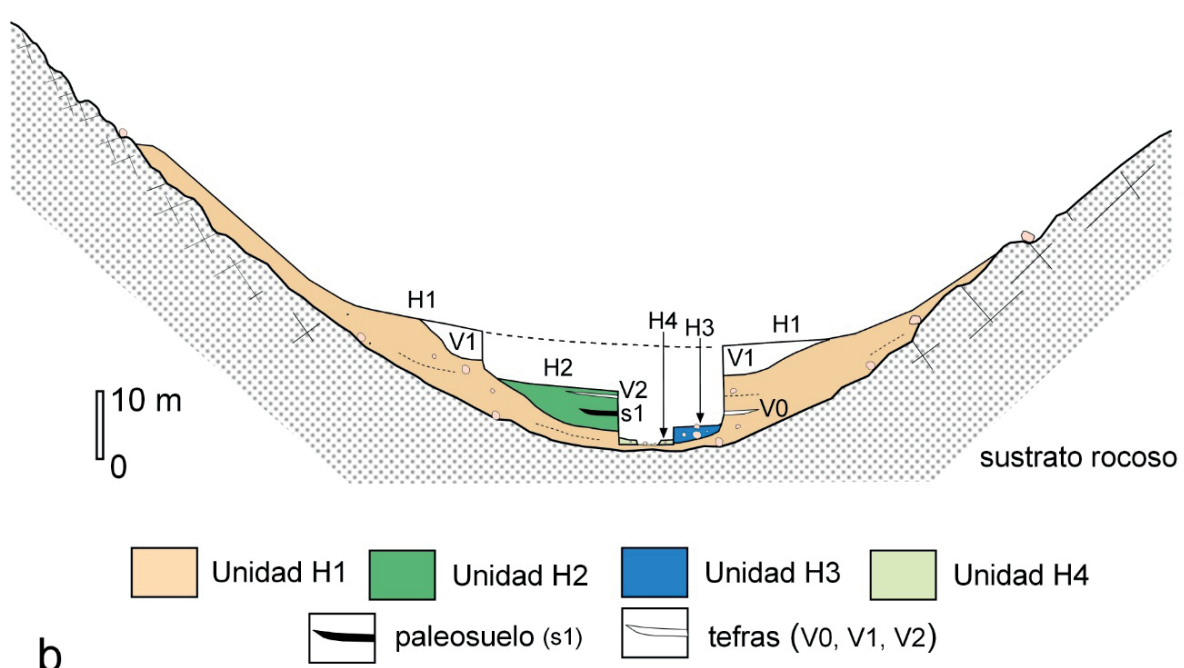

b

(1) zanjón 6

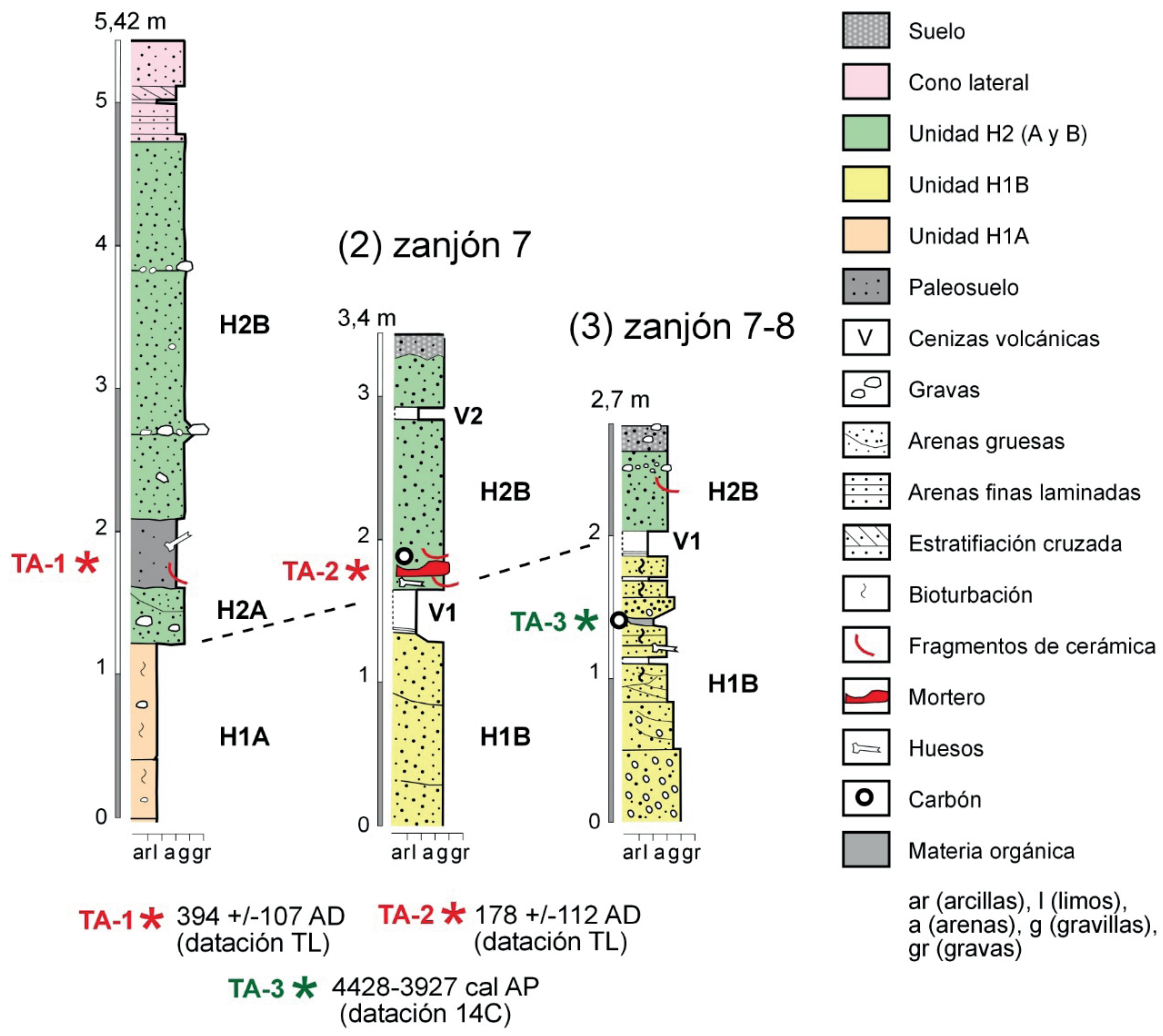

Figura 3. Información de escala intermedia. a) corte transversal de las unidades morfosedimentarias de un valle secundario, afluente del rio Tafi; b) correlación estratigráficas de las unidades en tres perfiles. Se han señalado las dataciones, los restos arqueológicos, el paleosuelo y las cenizas volcánicas (figura de los autores). 

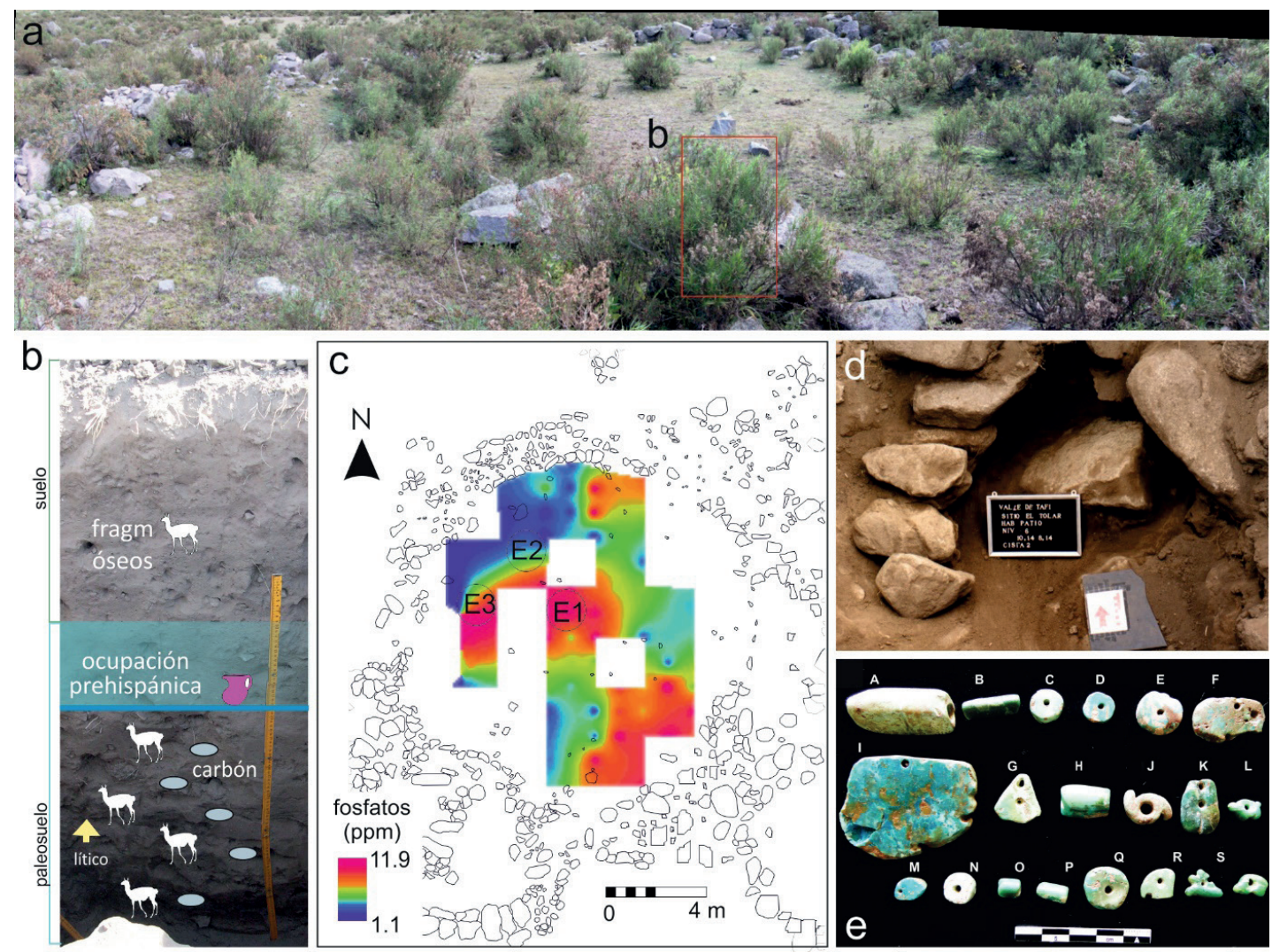

Figura 4. Información de escala de detalle: (a) estructura residencial en La Costa 2 (valle de Tafi) con zona de excavación indicada con «b»; (b) corte estratigráfico de la excavación; (c) unidad residencial excavada en El Tolar y determinación de áreas de concentración de fosfatos y distribución de entierros (E1 a E2); (d) entierro E2; (e) cuentas de collar producto de intercambios de larga distancia halladas en E2 (figura de los autores).

degradativos sobre suelos de la época se manifiestan en compactación, pérdida de materia orgánica, pérdida de porosidad, alteraciones químicas y erosión, identificados a partir de la descripción de perfiles de suelos y análisis fisicoquímicos de las muestras obtenidas, que afectan a todos los sectores productivos en conjunto (Sampietro-Vattuone et al. 2018a).

Siguiendo en una escala de detalle que permita completar el modelo geoarqueológico, se estudiaron las conexiones existentes entre las prácticas de subsistencia, el impacto de las mismas en suelos y paisaje y la vida cotidiana en unidades residenciales (Fig. 4a, 4b) en términos, por ejemplo, de áreas de actividad dentro de unidades residenciales (Fig. 4c) (Sampietro y Vattuone 2005), prácticas de almacenaje, prácticas inhumatorias (Fig. 4d, 4e) (Sampietro y Vattuone 2010) y cúlticas (Sampietro Vattuone et al. 2008) (Fig. 4e), con persistencia de ocupación de las estructuras residenciales (Cremonte 1988). Este último incluido en procesos de escala intermedia de duración representado por el tiempo de uso de cada unidad residencial, que ha demostrado alcanzar varios siglos, en contraste con las prácticas inhumatorias ocurridas en momentos acotado como hitos tanto en tiempo como en espacio - de la vida de la familia que habitaba cada unidad residencial específica. Más aún, la existencia de indicadores territoriales, representados por señas puntuales, como las estelas de piedra situadas en puntos geográficamente estratégicos (Sampietro-Vattuone et al. 2019), están marcando el enlace entre escalas de percepción y comprensión del entorno por 
parte de las sociedades pasadas del valle que son asimilables al esquema presentado. En el caso de estudio, representan un ejemplo de la escala de comprensión del territorio (enlazado con una escala de percepción regional, e incluso extra regional, al integrar los indicios de intercambio comercial de larga distancia) (Sampietro Vattuone et al. 2017) en un tiempo acotado de alta resolución.

La síntesis de todas estas evidencias articuladas temporal y espacialmente se recoge en el modelo evolutivo geoarqueológico del valle de Tafí, integrando diversas escalas — tanto espaciales como temporales - en un todo complejo y articulando información local, regional y global de los procesos identificados durante todo el Holoceno (Sampietro-Vattuone y Peńa-Monné 2016; Peña Monné y Sampietro Vattuone 2018). En este caso, presentamos el detalle correspondiente al Holoceno Superior (Fig. 5), los últimos tres milenios del modelo. En el mismo se puede apreciar las cuatro fases agradativas representadas en nuestro mapa ( $\mathrm{H} 1$ a $\mathrm{H} 4)$ que se produjeron a lo largo de los últimos 3000 años (Fig. 2). Los inicios de la unidad H2 presentan un ritmo de deposición lento, e incluso un período de estabilidad representado por un suelo datado hace alrededor de 2500 ańos de antigüedad, contemporáneo con un período húmedo a nivel mundial, denominado Evento Bond 2,8 (Bond et al. 1997). Este suelo fue la superficie sobre la cual se asentaron las primeras comunidades sedentarias del Formativo. A partir de este momento, la información morfoestratigráfica muestra una aceleración de los procesos de sedimentación sobre las unidades agradativas (Fig. 5). En escala de detalle este proceso es contemporáneo con los rasgos degradativos ya mencionados, que son preeminentes en las zonas de explotación agraria. Además es un proceso contemporáneo con el gradual incremento demográfico y de la presión de exploración sobre el ambiente. El período en conjunto incluye dos momentos de particular aridez identificada por diversos indicadores de la región: la Anomalíca Cálida Medieval hacia el 1000 AD y otro período particularmente árido alrededor del $1300 \mathrm{AD}$. Si se observa la señal radiocarbónica en la Fig. 4, puede notarse cómo en el lapso temporal comprendido entre ambas fases áridas, esta disminuye sustancialmente, habiendo una recuperación de la misma a partir de las fases húmedas de la Pequeña Edad del Hielo (Peña Monné y Sampietro Vattuone 2018; Sampietro Vattuone et al. 2018a). En conjunto, el modelo ha integrado múltiples escalas de análisis, tanto en términos espaciales como temporales. Su realización requiere necesariamente especialistas formados en el reconocimiento arqueológico y en la evolución del paisaje. En el caso presentado la investigación fue dirigida por una arqueóloga y un geomorfológo, con una sólida formación interdisciplinaria orientada a la geoarqueología, que incluye conocimientos de geología y suelos, así como de representación cartográfica, con el apoyo puntual de otros especialistas formados en métodos fisicoquímicos de análisis de suelos.

\section{Conclusiones}

La construcción de un modelo geoarqueológico de una región implica el manejo conjunto de diversas escalas temporales y espaciales, lo cual otorga riqueza a las interpretaciones y una perspectiva coevolutiva de las variables culturales y naturales, muchas veces interpretadas como opuestas y antagónicas. La escala regional se inicia con la generación de un mapa geomorfológico, que permite presentar el lienzo contextual sobre el cual interactuarán todas las variables que se irán registrando a partir de las diversas técnicas aplicadas. La imbricación de las mismas se impone como una necesidad para comprender la dinámica de un espacio en términos humanos y naturales, como fuerzas sinérgicas que modelan el paisaje que llega hasta nuestros días. La escala intermedia se asocia al estudio de morfologías, registros morfosedimentarios y los yacimientos en un contexto evolutivo más restringido y dentro de un marco cronológico definido. Por último, la escala de detalle promueve un análisis con mayor resolución de los sitios arqueológicos (incluyendo su cultura material), el uso del suelo agrario y la intervención en el territorio. 


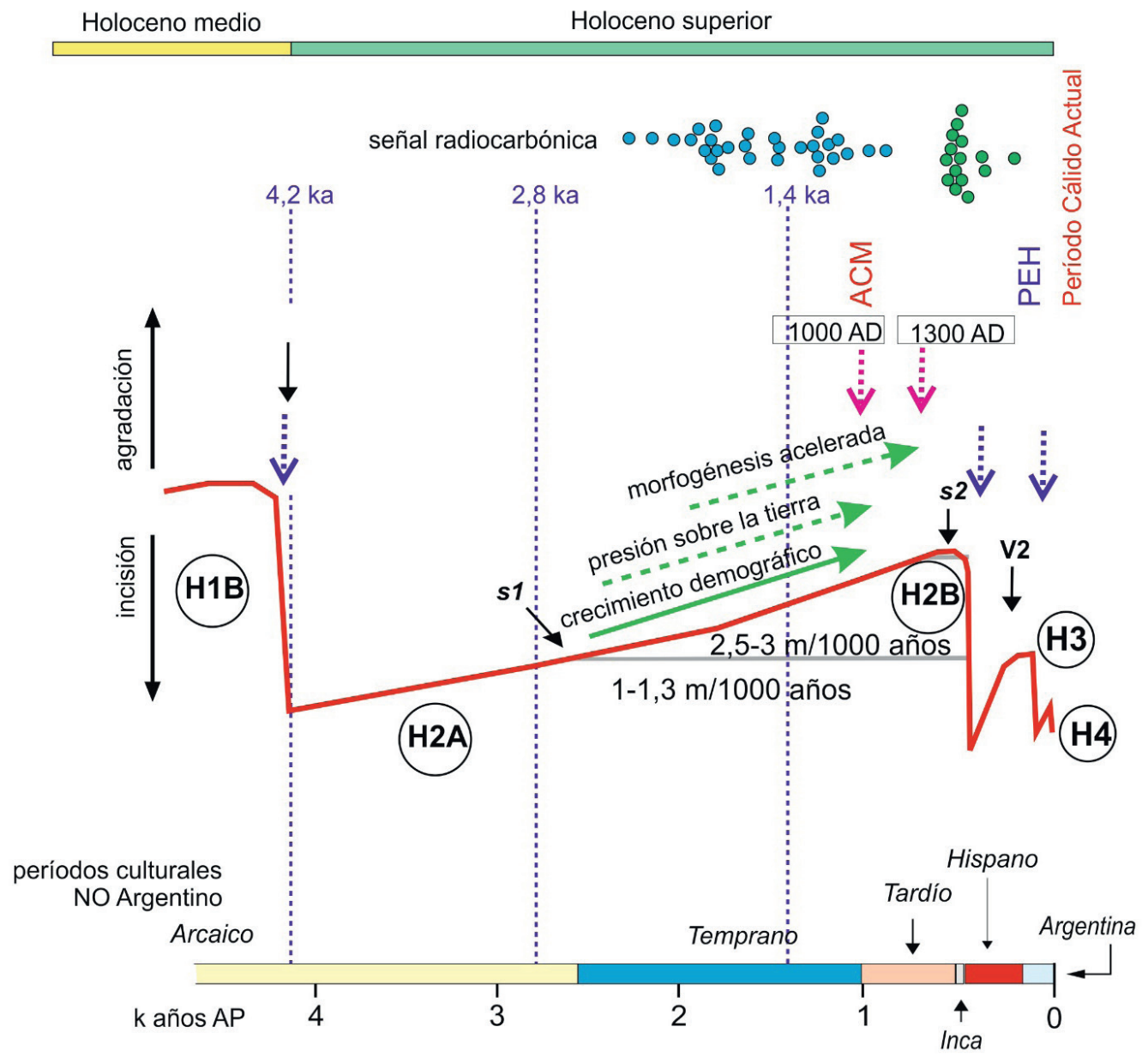

Figura 5. Modelo geoarqueológico del valle de Tafi durante el Holoceno Superior, integrando variables humanas y ambientales de carácter local, regional y global. Los números en color azul corresponden a los eventos Bond (Bond et al., 1997); las flechas azules son fases de incisión importantes; las flechas rojas son periodos secos constatados; s1, s2 paleosuelos; V2 nivel de tefra; las flechas verdes marcan la progresión de la intervención humana (figura de los autores).

La comprensión adecuada de los procesos, las escalas espaciotemporales implicadas y la calidad de las interpretaciones depende de las fuentes utilizadas y la adecuación de los métodos de aproximación. Por otra parte, la correcta interpretación del modelo geoarqueológico en cuanto a contenido y alcance permite entender su funcionamiento integrador como herramienta heurística de trabajo y promotora de explicaciones coherentes integradas de largo alcance.

\section{Agradecimientos}

Este trabajo es una contribución del Grupo de investigación «Primeros Pobladores del valle del Ebro» del Gobierno de Aragón, y el IUCA (Instituto de Ciencias Ambientales de la Universidad de Zaragoza, España) y subsidios PIUNT G629 de la Universidad Nacional de Tucumán y PICT2018-1119 y PICT2019-0931 de ANPCyT. 


\section{Referencias}

Alker, H. R. (1969). A Typology of Ecological Fallacies, en: M. Dogan y S. Rokkan (eds.), Quantitative Ecological Analysis in the Social Sciences, 69-86, Cambridge.

Banning, E. B. (2002). Archaeological survey (vol. 1), Springer Science \& Business Media.

Bird, J. H. (1989). The changing worlds of Geography: a critical guide to concepts and methods, Oxford University Press, New York.

Bond, G., W. Showers, M. Cheseby, R. Lotti, P. Almasi, P. deMenocal, P. Priore, H. Cullen, I. Hajdas, y G. Bonani (1997). A pervasive millennial scale cycle in north Atlantic Holocene and glacial climates, Science 278, $1257-1266$.

Burger, O. y L. C. Todd (2006). Grain, extent, and intensity: the components of scale in archaeological survey, en: G. Lock y B. Molyneaux (eds.), Confronting scale in archaeology, 235-255, Springer, Boston.

Burillo, F. y J. L. Peña Monné (1984). Clima, geomorfología y ocupación humana. Introducción a un planteamiento metodológico, en: Actas de las Primeras Jornadas de Metodología de Investigación Prehistórica (1981), 91-102, Soria.

Butzer, K. W. (2008). Challenges for a cross-disciplinary geoarchaeology: the intersection between environmental history and geomorphology, Geomorphology 101, 402-411.

Clarke, D. L. (1977). Spatial Archaeology, Academic Press, London.

Constante, A., J. L. Peña-Monné y A. Muñoz (2010). Alluvial geoarchaeology of an ephemeral stream: Implications for Holocene landscape change in the Central part of the Ebro Depression, Northeast Spain, Geoarchaeology 25(4), 475-496.

Constante, A., J. L. Peña-Monné, A. Muñoz, J. Picazo (2011). Climate and anthropogenic factors affecting alluvial fan development during the Late Holocene in the Central Ebro valley, Northeast Spain, Holocene 21, 275-286.

Cremonte, M. B. (1988). Comentario sobre fechados radiocarbónicos del sitio El Pedregal (Qda La Ciénega, Dto Tafi, Tucumán, Argentina), Chungará 23, 9-19.

Denham, T. (2008). Environmental archaeology: interpreting practices-in-the landscape through geoarchaeology, en: B. David (ed.), Handbook of Landscape Archaeology, 468-481, Thomas, Walnut Creek, California.

Edwards, T., E. Grono, A. I. Herries, F. J. Brink, U. Troitzsch, T. Senden, M. Turner, A. Barron, L. Prossor y T. Denham (2017). Visualising scales of process: Multi-scalar geoarchaeological investigations of microstratigraphy and diagenesis at hominin bearing sites in South African karst, Journal of Archaeological Science 83, 1-11.

Fouache, E. (2013). The geoarchaeological approach, en: C. Corsi, B. Splapšak y F. Vermeulen (eds.), Good Practice in Archaeological Diagnostics, 245-252, Springer International Publishing, Cham.

Gibson, J. J. (1977). The Theory of Affordances, en: R. Shaw y J. Bransford (eds.), Perceiving, Acting and Knowing: Toward an Ecological Psychology, 67-88, Erlbaum, Hillsdale, New Jersey.

Gibson, J. J. (1982). Notes on Affordances, en: E. Reed y R. Jones (eds.), Reasons for Realism, 401-418, Erlbaum, Hillsdale, New Jersey.

Haggett, P. (1965). Scale Components in Geographical Problems, en: R. J. Chorley y P. Haggett (eds.), Frontiers in Geographical Teaching, 164-185, Methuen, London.

Harris, T. M. (2006). Scale as artifact: GIS, ecological fallacy, and archaeological analysis, en: G. Lock y B. Molyneaux (eds.), Confronting scale in archaeology, 39-53, Springer, Boston.

Harvey, D. W. (1968). Pattern, Process and the Scale Problem in Geographical Research, Transactions of the Institute of British Geographers 45, 71-78.

Lock, G. y B. Molyneaux (2006). Introduction, en: G. Lock y B. Molyneaux (eds.), Confronting scale in archaeology, 1-13, Springer, Boston.

Maldonado, M. G., M. M. Sampietro Vattuone, A. M. Blasi, C. Castiñeira Latorre, J. L. Peña Monné y M. A. Pigoni (2016). Estratigrafía, paleoambiente y procesos de formación de sitio en El Paso durante el Holoceno tardío (valle de Santa María- Noroeste Argentino), en: M. M. Sampietro Vattuone y J. L. Peña Monné (eds.), Geoarqueología de los Valles Calchaquies, 121-144, Laboratorio de Geoarqueología, Universidad Nacional de Tucumán.

Mathieu, J. R. y R. E. Scott (eds.) (2004). Exploring the Role of Analytical Scale in Archaeological Interpretation, BAR International Series 1261, Oxford.

Mcmaster, R. B. y E. Sheppard (eds.) (2004). Scale and Geographic Inquiry: Nature, Society, and Method, Blackwell, Bodmin, Cornwall.

McNamee, C. y L. Wilson (2013). Multi-scalar approaches to geoarchaeological questions, Geoarchaeology 28(3), 191-194. 
Modderman, P. J. R. (1988). Thoughts on archaeology, geography and scales, en: J. L. Bintliff, D. A. Davidson y E. G. Grant (eds.), Conceptual issues in environmental archaeology, 59-63, Edinburgh University Press, Edinburgh.

Peña Monné, J. L. (2018). Geoarqueología aplicada a la reconstrucción paleoambiental: La evolución del Holoceno superior en el NE de España, Boletín Geológico y Minero 129 (1/2), 285-303.

Peña Monné, J. L. y M. M. Sampietro Vattuone (2018). Paleoambientes holocenos del valle de Tafí (Noroeste Argentino) a partir de registros morfosedimentarios y geoarqueológicos, Boletín Geológico y Minero 129(4), 671-691.

Peña Monné, J. L., A. Julián, J. Chueca, M.T. Echeverría y G. Ángeles (2004). Etapas de evolución holocena en el valle del río Huerva: Geomorfología y Geoarqueología, en: J. L. Peña-Monné, L.A. Longares Aladrén, M. Sánchez Fabre (eds.), Geografía Física de Aragón. Aspectos generales y temáticos, 289-302, Instituto Fernando el Católico, Universidad de Zaragoza, Zaragoza.

Peña Monné, J. L., J. R. González y J. I. Rodríguez (1996). Paleoambientes y evolución geomorfológica en yacimientos arqueológicos del sector oriental de la depresión del Ebro durante el Holoceno superior, en: A. PérezAlberti, P. Martini, W. Chesworth y A. Martínez-Cortizas (eds.), Dinámica y evolución de medios cuaternarios, 63-80, Xunta de Galicia.

Peña Monné, J. L., M. M. Sampietro Vattuone, J. V. Picazo, F. Pérez Lambán, L. A. Longares y D. Badia (2018b). Applications of geoarchaoelogical evolutionary models for the interpretation of complex archaeological structures in the central Ebro Basin (Spain), Quaternary International 483, 70-82.

Peña Monné, J. L., M. M. Sampietro Vattuone, L. A. Longares Aladrén, F. Pérez Lambán, M. Sánchez Fabre, M. Alcolea Gracia, L. Vallés, M. T. Echeverría y C. Baraza (2018a). Holocene alluvial sequence of Valderazagoza (Los Monegros) in the paleoenvironmental context of the Ebro Basin (Spain), Geographical Research Letters 44(1), 321-348.

Peña Monné, J. L., M. M. Sampietro Vattuone, M. G. Maldonado, S. F. Cano y M. Aguirre (2016). Contexto geomorfológico y problemas de conservación en el sitio arqueológico El Pichao (valle de Santa María, Noroeste Argentino), en: M. M. Sampietro Vattuone y J. L. Peña Monné (eds.), Geoarqueología de los Valles Calchaquies, 165-184, Laboratorio de Geoarqueología, Universidad Nacional de Tucumán, San Miguel de Tucumán.

Peña-Monné, J. L. y M. M. Sampietro Vattuone (2014). Geoarchaeological and paleoenvironmental reconstruction through evolutionary models: Dryland applications, European Geologist Magazine 38, 40-44.

Peña-Monné, J. L. y M. M. Sampietro-Vattuone (2019). Late Holocene anthropic degradation records in semiarid environments (NE Spain and NW Argentina), Geographical Research Letters 45(1), 195-217.

Peña-Monné, J. L., C. Sancho, V. Durán y R. Mikkan (2013). Geoarchaeological reconstruction of the Caverna de las Brujas (Mendoza, Argentina) for the planning of an archaeological intervention, Quaternary International 308-309, 265-272.

Peña-Monné, J.L., V. Rubio-Fernández, L. A. Longares-Aladrén, F. J. Gutiérrez-González, F. Pérez-Lambán y C. Laliena-Corbera (2014). The geomorphological context of Medieval Juslibol Castle in the middle reaches of the River Ebro, Spain, Geoarchaeology 29, 448-461.

Pérez-Lambán, F., J. L. Peña-Monné, J. Fanlo, J. V. Picazo, D. Badia, V. Rubio, R. García-Jiménez y M. M. Sampietro Vattuone (2014). Paleoenvironmental and geoarchaeological reconstruction from late Holocene slope records (Lower Huerva Valley, Ebro Basin, NE Spain), Quaternary Research 81, 1-14.

Rapp, G. (2007). Reconstructing Human-Landscape Interactions, Cambridge Scholars Publishing, Newcastle.

Robinson, W. S. (1950). Ecological Correlations and the Behavior of Individuals, American Sociological Review 15(3), 351-357.

Sampietro Vattuone M. M. y J. L. Peña Monné (2019a). Cambios ambientales y geoarqueología en medios áridos y semiáridos: Propuesta metodológica, Boletin de la Sociedad. Geológica. Mexicana 71(2), 565-584.

Sampietro Vattuone, M. M. (2010). Espacio, ambiente y los inicios de la agricultura indigena en el noroeste argentino: un enfoque geoarqueológico, JAS Arqueología, Madrid.

Sampietro Vattuone, M. M. y J. L. Peña-Monné (2014). Reconstruction of agrarian practice and land impact in the drylands: A geoarchaeological approach, European Geologist Magazine 38, 5-8.

Sampietro Vattuone, M. M. y J. L. Peña-Monné (2016). Geomorphological dynamic changes during the Holocene through ephemeral stream analyses from Northwest Argentina, Catena, 247, 663-677.

Sampietro Vattuone, M. M., J. L. Peña Monné, M. G. Maldonado, C. Sancho, W. Báez, A. Sola (2018b). Registro de cambios ambientales durante el Holoceno superior en depósitos fluvio-eólicos del Valle de Santa María (Noroeste Argentino), Boletín Geológico y Minero 129(4), 647-669.

Sampietro Vattuone, M. M., J. L. Peña-Monné, J. Roldán, M. G. Maldonado, M. G. Lefebvre, y M. A. Vattuone (2018a). Human-driven geomorphological processes and soil degradation in Northwest Argentina: a geoarchaeological view, Land Degradation and Development 29, 3852-3865. 
Sampietro Vattuone, M. M., L. Neder, J. Roldán y M. A. Vattuone (2008). Mother Earth: soil and people relationships during the prehispanic period (Northwest Argentina), World Archaeology 40(2), 190-205.

Sampietro Vattuone, M. M., S. Martínez Stagnaro, R. García Giménez, J. L. Peña Monné, J. Roldán y M. G. Maldonado (2017). Graves, beads, and trade in Northwest Argentina: a first ED-XRF characterization of very well-formed objects, Arqueología 23(1), 27-43.

Sampietro, M. M. y M. A. Vattuone (2005). Reconstruction of activity areas at a Formative household in northwest Argentina, Geoarchaeology 20(4), 337-354.

Sampietro-Vattuone, M. M. y J. L. Peña-Monné (2019b). Geomorphology of Tafi valley (Tucumán Province, Northwest Argentina), Journal of Maps 15(2), 177-184.

Sampietro-Vattuone, M. M., J. L. Peña Monné, J. Roldán, A. B. Dip, M. G. Maldonado, M. G. Lefebvre y M. A. Vattuone (2019). Land management and soil degradation evidence during the Late Holocene in Northwest Argentina (La Costa 2-Tafí valley), Catena, 182, 104-115.

Schumm, S. A. (1991). To interpret the Earth: Ten ways to be wrong, University Press, New York, Cambridge.

Smith, N. (2000). Scale, en: R. J. Johnston, D. Gregory, G. Pratt y M.Watts (eds.), The Dictionary of Human Geography, $4^{\text {th }}$ ed., 457-458, Blackwell, Oxford.

Spaulding, A. C. (1960). Statistical description and comparison of artifact assemblages, Bobbs-Merrill, Indianapolis.

Stein, G. J. (2002). From passive periphery to active agents: emerging perspectives in the archaeology of interregional interaction, American Anthropologist 104(3), 903-916.

Stein, J. K. (1993). Scale in archaeology, geosciences, and geoarchaeology, en: J. K. Stein, y A. R. Linse (eds.), Effects of Scale on Archaeological and Geoscientific Perspectives, vol. 283, 1-10, Geological Society of America, Boulder.

Webb, J. W. (1976). Geographers and scale, en: L. A. Kosinske y J. W. Webb (eds.), Population at microscale: Palmerston North, New Zealand, 13-19, Massey University, Commission of Population Geography, New Zealand Geographical Society.

Wobst, H. M. (2006). Artifacts as social interference: The politics of spatial scale, en: G. Lock y B. Molyneaux (eds.), Confronting scale in archaeology, 55-64. Springer, Boston.

Zimmerman, L. J. y J. A. Artz (2006). Scale, model complexity, and understanding: simulation of settlement processes in the Glenwood locality of Southwestern Iowa, 1976 and 2000, en: G. Lock y B. Molyneaux (eds.), Confronting scale in archaeology, 129-144. Springer, Boston.

Recibido: mayo 2021

Aceptado: mayo 2021 